QUANTUM DYNAMICS

BANACH CENTER PUBLICATIONS, VOLUME 120
INSTITUTE OF MATHEMATICS
POLISH ACADEMY OF SCIENCES
WARSZAWA 2020

\title{
A SPECTRAL TRIPLE FOR NONCOMMUTATIVE COMPACT SURFACES
}

\author{
FREDY DÍAZ GARCÍA and ELMAR WAGNER \\ Instituto de Física y Matemáticas, Universidad Michoacana de San Nicolás de Hidalgo \\ Cd. Universitaria, Edificio C-3, 58040 Morelia, Michoacán, México \\ ORCID: 0000-0001-6055-701X, 0000-0003-3932-9332 \\ E-mail: lenonndiaz@gmail.com,elmar@ifm.umich.mx
}

Dedicated to Paul Baum on the occasion of his 80th birthday who taught me like nobody else that teaching difficult mathematics doesn't have to be difficult and can be fun.

\begin{abstract}
A Dirac operator is presented that will yield a $1^{+}$-summable regular even spectral triple for all noncommutative compact surfaces defined as subalgebras of the Toeplitz algebra. Connes' conditions for noncommutative spin geometries are analyzed and it is argued that the failure of some requirements is mainly due to a wrong choice of a noncommutative spin bundle.
\end{abstract}

1. Introduction. One of the most fundamental notions of Connes' noncommutative geometry [2] is that of a spectral triple. An extensive amount of research has been dedicated to finding novel examples. However, proving that a spectral triple satisfies all requirements of a noncommutative spin geometry can be a difficult task. Among the noteworthy examples are the noncommutative torus which satisfies all proposed conditions [4, the isospectral deformations of toric manifolds [6], and the standard Podleś sphere [9]. In the latter case, the original conditions had to be modified to conform with the basic principles of a noncommutative spin geometry [14. This seems to be a common feature for examples arising in quantum group theory. In particular, for the natural spectral triple on quantum $\mathrm{SU}(2)$ [8], it was proven that the requirement of a real structure demands a modification of original framework. A proposal for such a modification can be found in [1].

2010 Mathematics Subject Classification: 58B34, 46L87.

Key words and phrases: noncommutative surfaces, Toeplitz algebra, spectral triple. The paper is in final form and no version of it will be published elsewhere. 
In this paper, we present a $1^{+}$-summable regular even spectral triple for the noncommutative compact surfaces from [15] and study it in the context of Connes' noncommutative spin geometry [11. It turns out that the same Dirac operator can be used for all noncommutative compact surfaces including the non-orientable ones. This already indicates that these spectral triples are not very useful for the calculation of topological invariants (yet this could be a welcome effect: if all quantum spaces behaved like the classical ones, there were no need for a quantization). The reason is that the spectral triples are defined on the "wrong" Hilbert space, namely on the holomorphic functions of the Bergman space on the unit disc rather than on sections of the noncommutative spin bundle. Although this seems obvious, such effects could easily be disguised in more complicated examples, especially if the finiteness and the regularity conditions can be satisfied as in our case. Under more favorable conditions, the finiteness and the regularity conditions lead the correct module related to the spin bundle. Here, however, as a consequence of working on the "wrong" Hilbert space, the spectrum of the Dirac operator resembles that of a 1-dimensional spectral triple and so does a possible real structure. Furthermore, the first order condition can only be satisfied up to compacts whereas the orientation condition and the Poincaré duality fail. In this sense, our paper is rather a warning that modifying or not fulfilling some axiomatic conditions may have substantial effects on the noncommutative spin geometry.

2. Noncommutative compact surfaces. The noncommutative compact surfaces of any genus [15] will be defined as subalgebras of the continuous functions on the quantum disc. As explained in [12], the universal $\mathrm{C}^{*}$-algebra of the quantum disc can be represented by the Toeplitz algebra. For a description of the Toeplitz algebra, consider the open complex unit disc $\mathbb{D}:=\{z \in \mathbb{C}:|z|<1\}$ with the standard Lebesgue measure and let $\overline{\mathbb{D}}:=\{z \in \mathbb{C}:|z| \leq 1\}$ denote its closure in $\mathbb{C}$. We write $L_{2}(\mathbb{D})$ for the Hilbert space of square-integrable functions and $A_{2}(\mathbb{D})$ for the closed subspace of holomorphic functions on $\mathbb{D}$. Let $\hat{P}$ denote the orthogonal projection from $L_{2}(\mathbb{D})$ onto $A_{2}(\mathbb{D})$. The Toeplitz operator $\hat{T}_{f} \in B\left(A_{2}(\mathbb{D})\right)$ with symbol $f \in C(\overline{\mathbb{D}})$ is defined by

$$
\hat{T}_{f}(\psi):=\hat{P}(f \psi), \quad \psi \in A_{2}(\mathbb{D}) \subset L_{2}(\mathbb{D}),
$$

and the Toeplitz algebra $\mathcal{T}$ is the $\mathrm{C}^{*}$-algebra generated by all $\hat{T}_{f}$ in $B\left(A_{2}(\mathbb{D})\right)$. As is well known [13, the compact operators $K\left(\ell_{2}(\mathbb{N})\right) \cong K\left(A_{2}(\mathbb{D})\right)$ belong to $\mathcal{T}$ and the quotient $\mathcal{T} / K\left(\ell_{2}(\mathbb{N})\right) \cong C\left(\mathbb{S}^{1}\right)$ gives rise to the $\mathrm{C}^{*}$-algebra extension

$$
0 \longrightarrow K\left(\ell_{2}(\mathbb{N})\right) \longrightarrow \mathcal{T} \stackrel{\hat{\sigma}}{\longrightarrow} C\left(\mathbb{S}^{1}\right) \longrightarrow 0,
$$

with the symbol map $\hat{\sigma}: \mathcal{T} \longrightarrow C\left(\mathbb{S}^{1}\right)$ given by $\hat{\sigma}\left(\hat{T}_{f}\right)=\left.f\right|_{\mathbb{S}^{1}}$ for all $f \in C(\overline{\mathbb{D}})$.

Recall that each closed surface can be constructed from a convex polygon by a suitable identification of its edges. Instead of edges of a polygon, we will consider arcs on the boundary of the unit disc. In this manner, the $\mathrm{C}^{*}$-algebra of continuous functions on a closed surface can be viewed as a subalgebra of $C(\overline{\mathbb{D}})$. Identifying points on the boundary means that the functions belonging to the specified subalgebra must have the same values on identified points. In correspondence with the presentation of oriented closed surfaces, 
let $g \in \mathbb{N}$ and define $4 g$ arcs on the circle $\mathbb{S}^{1}$ by

$$
\begin{aligned}
a_{k}, a_{k}^{-1}:[0,1] \longrightarrow \mathbb{S}^{1}, \quad & a_{k}(t):=\mathrm{e}^{\pi \mathrm{i}(k-1+t) /(2 g)}, \\
& a_{k}^{-1}(t):=\mathrm{e}^{\pi \mathrm{i}(2 g+k-t) /(2 g)}, \quad k=1, \ldots, 2 g .
\end{aligned}
$$

By standard operations from algebraic topology, one can readily show that

$$
\mathbb{T}_{g}:=\overline{\mathbb{D}} / \sim, \quad z \sim z \quad \text { and } \quad a_{k}(t) \sim a_{k}^{-1}(t) \text { for all } z \in \mathbb{D}, t \in[0,1], k=1, \ldots, 2 g,
$$

is homeomorphic to a closed oriented surface of genus $g$.

Viewing the symbol map $\hat{\sigma}: \mathcal{T} \rightarrow C\left(\mathbb{S}^{1}\right)$ as the counterpart of an embedding of the circle into the quantum disc, we define

$$
C\left(\mathbb{T}_{g, q}\right):=\left\{f \in \mathcal{T}: \hat{\sigma}(f)\left(a_{k}(t)\right)=\hat{\sigma}(f)\left(a_{k}^{-1}(t)\right) \text { for all } t \in[0,1], k=1, \ldots, 2 g\right\},
$$

see [15. To include the 2 -sphere with genus 0 , we consider additionally

$$
a_{0}, a_{0}^{-1}:[0,1] \longrightarrow \mathbb{S}^{1}, \quad a_{0}(t):=\mathrm{e}^{\pi \mathrm{i} t}, \quad a_{0}^{-1}(t):=\mathrm{e}^{-\pi \mathrm{i} t} .
$$

and define $C\left(\mathbb{S}_{q}^{2}\right):=C\left(\mathbb{T}_{0, q}\right)$ as in (2) with $k=0$. Similarly, for non-orientable closed surfaces, let

$$
a_{k}, b_{k}:[0,1] \longrightarrow \mathbb{S}^{1}, \quad a_{k}(t):=\mathrm{e}^{\pi \mathrm{i}(k-1+t) / g}, \quad b_{k}(t):=\mathrm{e}^{\pi \mathrm{i}(-k+t) / g}, \quad k=1, \ldots, g,
$$

and set

$$
C\left(\mathbb{P}_{g, q}\right):=\left\{f \in \mathcal{T}: \hat{\sigma}(f)\left(a_{k}(t)\right)=\hat{\sigma}(f)\left(b_{k}(t)\right) \text { for all } t \in[0,1], k=1, \ldots, g\right\} .
$$

From the continuity of the symbol map, it follows that $C\left(\mathbb{T}_{g, q}\right)$ and $C\left(\mathbb{P}_{g, q}\right)$ are $\mathrm{C}^{*}$-subalgebras of $\mathcal{T}$.

An alternative, albeit less illustrative description of these $\mathrm{C}^{*}$-algebras can be given by considering $L_{2}\left(\mathbb{S}^{1}\right)$ with orthonormal basis $\left\{e_{k}:=\frac{1}{\sqrt{2 \pi}} u^{k}: k \in \mathbb{Z}\right\}$, where $u\left(\mathrm{e}^{\mathrm{i} t}\right)=\mathrm{e}^{\mathrm{i} t}$ denotes the unitary generator of $C\left(\mathbb{S}^{1}\right) \subset L_{2}\left(\mathbb{S}^{1}\right)$. Let $P$ denote the orthogonal projection from $L_{2}\left(\mathbb{S}^{1}\right)$ onto the closed subspace $\ell_{2}(\mathbb{N}):=\overline{\operatorname{span}}\left\{e_{n}: n \in \mathbb{N}_{0}\right\}$. Then the Toeplitz algebra $\mathcal{T} \subset B\left(\ell_{2}(\mathbb{N})\right)$ is generated by the operators $T_{f}, f \in C\left(\mathbb{S}^{1}\right)$, where

$$
T_{f}(\phi):=P(f \phi), \quad \phi \in \overline{\operatorname{span}}\left\{e_{n}: n \in \mathbb{N}_{0}\right\} \subset L_{2}\left(\mathbb{S}^{1}\right),
$$

and the symbol map $\sigma: \mathcal{T} \rightarrow C\left(\mathbb{S}^{1}\right)$ reads $\sigma\left(T_{f}\right)=f$.

On the topological side, the $\mathrm{C}^{*}$-algebra of continuous functions on $\mathbb{S}^{1}$ satisfying the conditions on $\hat{\sigma}(f)$ in $[2]$ is isomorphic to $C\left(\bigvee_{k=1}^{2 g} \mathbb{S}^{1}\right)$, where $\bigvee_{k=1}^{2 g} \mathbb{S}^{1}$ denotes the topological wedge product of $n$ circles. Indicating the orientation of the identified arcs with a + or a - , we set

$$
\begin{aligned}
C\left(\bigvee^{2 g} \mathbb{S}^{1}\right) & :=\left\{f \in C\left(\mathbb{S}^{1}\right): f\left(a_{k}(t)\right)=f\left(a_{k}^{-1}(t)\right) \text { for all } t \in[0,1], k=1, \ldots, 2 g\right\}, \\
C\left(\forall \mathbb{S}^{1}\right) & :=\left\{f \in C\left(\mathbb{S}^{1}\right): f\left(a_{k}(t)\right)=f\left(b_{k}(t)\right) \text { for all } t \in[0,1], k=1, \ldots, g\right\} .
\end{aligned}
$$

Then the definitions of $C\left(\mathbb{T}_{g, q}\right)$ and $C\left(\mathbb{P}_{g, q}\right)$ can be rewritten as

$$
C\left(\mathbb{T}_{g, q}\right)=\left\{f \in \mathcal{T}: \sigma(f) \in C\left(\bigvee^{2 g} \mathbb{S}^{1}\right)\right\}, \quad C\left(\mathbb{P}_{g, q}\right)=\left\{f \in \mathcal{T}: \sigma(f) \in C\left(\forall^{g} \mathbb{S}^{1}\right)\right\} .
$$

By the short exact sequence 11 and the definitions of $C\left(\mathbb{T}_{g, q}\right)$ and $C\left(\mathbb{P}_{g, q}\right)$, these $\mathrm{C}^{*}$-algebras contain obviously $K\left(\ell_{2}(\mathbb{N})\right)=\operatorname{ker}(\sigma)$. Restricting the symbol map to $C\left(\mathbb{T}_{g, q}\right)$ 
and $C\left(\mathbb{P}_{g, q}\right)$ yields the $\mathrm{C}^{*}$-extensions

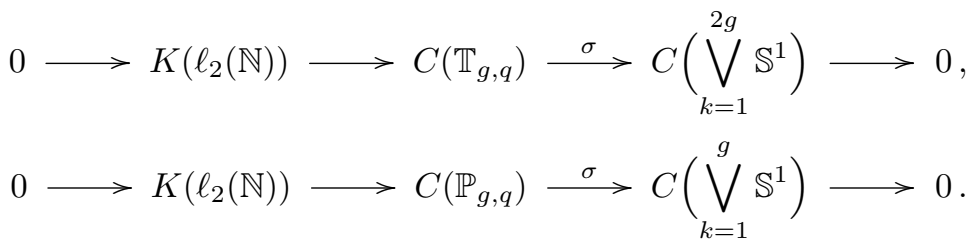

The $\mathrm{C}^{*}$-algebra extensions (6) provide a computational tool for calculating the $K$-groups of the noncommutative compact surfaces. The result can be found in [15] and is given by

$$
\begin{aligned}
K_{0}\left(C\left(\mathbb{T}_{g, q}\right)\right) \cong \mathbb{Z} \oplus \mathbb{Z}, \quad K_{1}\left(C\left(\mathbb{T}_{g, q}\right)\right) \cong \bigoplus_{k=1}^{2 g} \mathbb{Z}, \\
K_{0}\left(C\left(\mathbb{P}_{g, q}\right)\right) \cong \mathbb{Z}_{2} \oplus \mathbb{Z}, \quad K_{1}\left(C\left(\mathbb{P}_{g, q}\right)\right) \cong \bigoplus_{k=1}^{g-1} \mathbb{Z} .
\end{aligned}
$$

Moreover, a set of free generators for $K_{0}\left(C\left(\mathbb{T}_{g, q}\right)\right)$ is given by the trivial projection $[1] \in K_{0}\left(C\left(\mathbb{T}_{g, q}\right)\right)$ and $\left[p_{e_{0}}\right] \in K_{0}\left(K\left(\ell_{2}(\mathbb{N})\right)\right) \hookrightarrow K_{0}\left(C\left(\mathbb{T}_{g, q}\right)\right)$, where $p_{e_{0}}$ denotes the 1-dimensional projection onto $\mathbb{C} e_{0}$. Note that the $K$-groups coincide with those of the classical counterparts but the function algebras $C\left(\mathbb{T}_{g}\right)$ and $C\left(\mathbb{P}_{g}\right)$ do not contain nontrivial $1 \times 1$-projections.

\section{Differential geometry of noncommutative compact surfaces}

3.1. Spectral triples and regularity. Since surfaces are even dimensional, we are looking for even spectral triples $(\mathcal{A}, \mathcal{H}, D, \gamma)$ for our noncommutative compact surfaces, i.e., a dense ${ }^{*}$-subalgebra $\mathcal{A}$ of $C\left(\mathbb{T}_{g, q}\right)$ (or $\left.C\left(\mathbb{P}_{g, q}\right)\right)$ which is stable under holomorphic functional calculus, a faithful representation $\pi: \mathcal{A} \rightarrow B(\mathcal{H})$, a self-adjoint operator $D$ on $\mathcal{H}$ with compact resolvent and a self-adjoint grading operator $\gamma$ satisfying $\gamma^{2}=\mathrm{id}$, $\gamma D=-D \gamma, \gamma \pi(a)=\pi(a) \gamma$ and $[D, \pi(a)]:=D \pi(a)-\pi(a) D \in B(\mathcal{H})$ for all $a \in \mathcal{A}$.

We say that a spectral triple is $n^{+}$-summable if $(1+|D|)^{-(n+\epsilon)}$ yields a trace class operator for all $\epsilon>0$ but $(1+|D|)^{-n}$ does not. In this case, one refers to the number $n \in[0, \infty)$ as the metric dimension in analogy to Weyl's formula for the asymptotic behavior of the eigenvalues of the Laplacian on a compact Riemannian manifold.

A spectral triple $(\mathcal{A}, \mathcal{H}, D)$ is called regular, if $\pi(a),[D, \pi(a)]) \in \bigcap_{n \in \mathbb{N}} \operatorname{dom}\left(\delta_{|D|}^{n}\right)$ for all $a \in \mathcal{A}$, where $\delta_{|D|}(x):=[|D|, x]$ and $\operatorname{dom}\left(\delta_{|D|}\right):=\left\{x \in B(\mathcal{H}): \overline{\delta_{|D|}(x)} \in B(\mathcal{H})\right\}$. Let $D=F|D|$ denote the polar decomposition of $D$. For regular even spectral triples, one can show that $F=\left(\begin{array}{cc}0 & F_{+-} \\ F_{-+} & 0\end{array}\right)$ provides an even Fredholm module for $\mathcal{A}$ and one defines $\operatorname{ind}(D):=\operatorname{ind}\left(F_{+-}\right)$. This Fredholm module is called the fundamental class of $D$ and we say that the fundamental class is non-trivial if it gives rise to non-trivial index pairings.

Before turning our attention to spectral triples for $C\left(\mathbb{T}_{g, q}\right)$, we will describe more explicitly the action of $\mathcal{T} \subset B\left(\ell_{2}(\mathbb{N})\right)$ on $\ell_{2}(\mathbb{N})$. As in the previous section, we use the orthonormal basis $\left\{e_{k}=\frac{1}{\sqrt{2 \pi}} u^{k}: k \in \mathbb{Z}\right\}$ of $L_{2}\left(\mathbb{S}^{1}\right)$. Then $T_{u^{n}}=S^{n}$ and $T_{u^{-n}}=S^{* n}$, 
where $S \in B\left(\ell_{2}(\mathbb{N})\right)$ denotes the shift operator given by

$$
S e_{k}=e_{k+1}, \quad k \in \mathbb{N}_{0} .
$$

Expanding $f \in C\left(\mathbb{S}^{1}\right) \subset L_{2}\left(\mathbb{S}^{1}\right)$ in its Fourier series $f=\sum_{k \in \mathbb{Z}} f_{k} u^{k}, f_{k}=\frac{1}{\sqrt{2 \pi}}\left\langle e_{k}, f\right\rangle$, and setting

$$
S^{\# k}:=S^{k}, \quad k \geq 0, \quad S^{\# k}:=S^{*|k|}, \quad k<0
$$

we can write

$$
T_{f}=\sum_{k \in \mathbb{Z}} f_{k} S^{\# k}
$$

which means that $T_{f} e_{n}=\sum_{k \in \mathbb{N}_{0}} f_{k-n} e_{k}$. Identifying $C^{(m)}\left(\mathbb{S}^{1}\right)$ with the space of $m$-times continuously differentiable $2 \pi$-periodic functions on $\mathbb{R}$, one shows by partial integration that

$$
T_{f^{\prime}}=\mathrm{i} \sum_{k \in \mathbb{Z}} k f_{k} S^{\# k}, \quad f \in C^{(1)}\left(\mathbb{S}^{1}\right),
$$

where $f^{\prime}=\frac{\mathrm{d}}{\mathrm{d} t} f$ for $t \in[0,2 \pi]$. In particular, $f=\sum_{k \in \mathbb{Z}} f_{k} u^{k} \in C^{\infty}\left(\mathbb{S}^{1}\right)$ implies that $\left\{f_{k}\right\}_{k \in \mathbb{Z}}$ is a sequence of rapid decay.

The condition that $\mathcal{A}$ should be stable under holomorphic functional calculus is often ignored because the proof that a chosen subalgebra has this property might be somewhat involved. Our choice is presented in the next proposition. The proof follows the arguments of [5, Proposition 1].

Proposition 3.1. Let $\mathcal{K}_{S} \subset K\left(\ell_{2}(\mathbb{N})\right)$ denote the ideal of matrices of rapid decay, i.e., operators $A$ given by $A e_{n}=\sum_{k \in \mathbb{N}_{0}} a_{k n} e_{k}$ such that $\lim _{(k, n) \rightarrow \infty}\left|k^{\alpha} a_{k n} n^{\beta}\right|=0$ for all $\alpha, \beta \in \mathbb{N}_{0}$. Set $C^{\infty}\left(\bigvee^{2 g} \mathbb{S}^{1}\right):=C^{\infty}\left(\mathbb{S}^{1}\right) \cap C\left(\bigvee^{2 g} \mathbb{S}^{1}\right)$ and let $\mathcal{A}$ be the ${ }^{*}$-algebra generated by the elements of $\mathcal{K}_{S}$ and $\mathcal{C}^{\infty}\left(\mathbb{T}_{g, q}\right):=\left\{T_{f} \in C\left(\mathbb{T}_{g, q}\right): f \in C^{\infty}\left(\bigvee^{2 g} \mathbb{S}^{1}\right)\right\}$. Then $\mathcal{A}$ is dense in $C\left(\mathbb{T}_{g, q}\right)$ and stable under holomorphic functional calculus.

Proof. Note that $\left\|T_{f}-T_{g}\right\| \leq\|P\|\|f-g\|_{\infty} \leq\|f-g\|_{\infty}$ and $x=x-T_{\sigma(x)}+T_{\sigma(x)}$ with $x-T_{\sigma(x)} \in K\left(\ell_{2}(\mathbb{N})\right)$. Since each $g \in C\left(\bigvee^{2 g} \mathbb{S}^{1}\right)$ can be uniformly approximated by functions from $C^{\infty}\left(\bigvee^{2 g} \mathbb{S}^{1}\right)$, and each compact operator can be approximated by matrices of rapid decay, $\mathcal{A}$ is dense in $C\left(\mathbb{T}_{g, q}\right)$.

An elementary calculation shows that

$$
\begin{aligned}
& S^{\#(m+k)}-S^{\# m} S^{\# k}=\left(1-S^{m} S^{* m}\right) S^{*(|k|-m)}\left(1-S^{|k|} S^{*|k|}\right), \quad k<0,0<m<|k|, \\
& S^{\#(m+k)}-S^{\# m} S^{\# k}=\left(1-S^{m} S^{* m}\right) S^{(m-|k|)}\left(1-S^{|k|} S^{*|k|}\right), \quad k<0, m \geq|k|,
\end{aligned}
$$

and 0 otherwise, where the operators in 10 are finite matrices. Let $f, g \in C^{\infty}\left(\bigvee^{2 g} \mathbb{S}^{1}\right)$. Using the representation (8) together with equation 10 and the fact that the coefficients are sequences of rapid decay, one proves that

$$
T_{f g}-T_{f} T_{g}=\sum_{m, k \in \mathbb{Z}} f_{m} g_{k}\left(S^{\# m+k}-S^{\# m} S^{\# k}\right) \in \mathcal{K}_{S} .
$$

Thus $\left[T_{f}, T_{g}\right] \in \mathcal{K}_{S}$, so the algebra generated by $\mathcal{C}^{\infty}\left(\mathbb{T}_{g, q}\right)$ is commutative modulo the ideal $\mathcal{K}_{S}$. Since $\mathcal{K}_{S}$ is an two-sided ideal in $\mathcal{A}$, we get the exact sequence

$$
0 \longrightarrow \mathcal{K}_{S} \longrightarrow \mathcal{A} \stackrel{\sigma}{\longrightarrow} C^{\infty}\left(\bigvee^{2 g} \mathbb{S}^{1}\right) \longrightarrow 0 .
$$


Moreover, $\mathcal{K}_{S}$ and $C^{\infty}\left(\bigvee^{2 g} \mathbb{S}^{1}\right)$ with the usual (semi-)norms for sequences of rapid decay and $C^{\infty}$-functions, respectively, can be turned into Fréchet algebras which are stable under holomorphic functional calculus. To prove the proposition, it thus suffices to show that, for all $a \in \mathcal{A}$ such that $a^{-1} \in C\left(\mathbb{T}_{g, q}\right)$, one has $a^{-1} \in \mathcal{A}$.

Now, if $a \in \mathcal{A}$ such that $a^{-1} \in C\left(\mathbb{T}_{g, q}\right)$, then clearly $\sigma\left(a^{-1}\right)=\sigma(a)^{-1} \in C^{\infty}\left(\bigvee^{2 g} \mathbb{S}^{1}\right)$. Furthermore, $1-a T_{\sigma\left(a^{-1}\right)} \in \mathcal{A}$ and $\sigma\left(1-a T_{\sigma\left(a^{-1}\right)}\right)=0$, hence $1-a T_{\sigma\left(a^{-1}\right)} \in \mathcal{K}_{S}$. As $\mathcal{K}_{S}$ is a two-sided ideal in $C\left(\mathbb{T}_{g, q}\right)$, we obtain $a^{-1}=a^{-1}\left(1-a T_{\sigma\left(a^{-1}\right)}\right)+T_{\sigma\left(a^{-1}\right)} \in \mathcal{A}$. This finishes the proof.

As also in the classical case not all continuous functions are differentiable, the last proposition provides us with a preferred choice of a dense ${ }^{*}$-subalgebra of $C\left(\mathbb{T}_{g, q}\right)$ for the construction of a spectral triple. In [10, a spectral triple for the noncommutative torus $C\left(\mathbb{T}_{1, q}\right)$ was obtained by starting with the action of the first order differential operator $\frac{\partial}{\partial z}$ on the Bergman space $A_{2}(\mathbb{D})$. It turns out that essentially the same Dirac operator works for all the noncommutative compact surfaces from Section 2 . In the following, we will first give the definition of these spectral triples and then prove their fundamental properties in a theorem.

The simplest choice of a $\mathbb{Z}_{2}$-graded Hilbert space with a faithful representation of $C\left(\mathbb{T}_{g, q}\right) \subset B\left(\ell_{2}(\mathbb{N})\right)$ is $\mathcal{H}:=\ell_{2}(\mathbb{N}) \oplus \ell_{2}(\mathbb{N})$. Then the assignment $\pi: \mathcal{A} \rightarrow B\left(\ell_{2}(\mathbb{N}) \oplus \ell_{2}(\mathbb{N})\right)$,

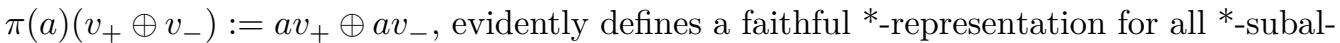
gebras $\mathcal{A} \subset B\left(\ell_{2}(\mathbb{N})\right)$. The representation commutes with the obvious grading operator $\gamma$ given by $\gamma\left(v_{+} \oplus v_{-}\right)=v_{+} \oplus\left(-v_{-}\right)$. Consider now the self-adjoint number operator $N$ defined by

$$
N e_{n}=n e_{n}, \quad \operatorname{dom}(N)=\left\{\sum_{n \in \mathbb{N}_{0}} \alpha_{n} e_{n} \in \ell_{2}(\mathbb{N}): \sum_{n \in \mathbb{N}_{0}} n^{2}\left|\alpha_{n}\right|^{2}<\infty\right\},
$$

and let

$$
D:=\left(\begin{array}{cc}
0 & S^{*} N \\
N S & 0
\end{array}\right)=\left(\begin{array}{cc}
0 & S^{*} N \\
S(N+1) & 0
\end{array}\right), \quad \operatorname{dom}(D)=\operatorname{dom}(N) \oplus \operatorname{dom}(N) \subset \mathcal{H} .
$$

Since $\left(S^{*} N\right)^{*}=N S=S(N+1), D$ is self-adjoint. Defining $F$ and $|D|$ by the polar decomposition $D=F|D|$, we get from 13 .

$$
F=\left(\begin{array}{cc}
0 & S^{*} \\
S & 0
\end{array}\right), \quad|D|=\left(\begin{array}{cc}
N+1 & 0 \\
0 & N
\end{array}\right) .
$$

Clearly, $\gamma D=-D \gamma$.

TheOrem 3.2. Let $\mathcal{A} \subset C\left(\mathbb{T}_{g, q}\right)$ denote the pre- $C^{*}$-algebra from Proposition 3.1, and let $\mathcal{H}, D, \gamma$ and the representation $\pi: \mathcal{A} \rightarrow B(\mathcal{H})$ be given as in the previous paragraph. Then $(\mathcal{A}, \mathcal{H}, D, \gamma)$ yields a $1^{+}$-summable regular even spectral triple for $C\left(\mathbb{T}_{g, q}\right)$. The Dirac operator $D$ has discrete spectrum $\operatorname{spec}(D)=\mathbb{Z}$, each eigenvalue $k \in \mathbb{Z}$ has multiplicity 1 and a complete orthonormal basis of associated eigenvectors is given by

$$
b_{k}:=\frac{1}{\sqrt{2}}\left(e_{k-1} \oplus e_{k}\right), \quad b_{-k}:=\frac{1}{\sqrt{2}}\left(-e_{k-1} \oplus e_{k}\right), \quad k>0, \quad b_{0}:=0 \oplus e_{0} .
$$

Moreover, the fundamental class of $D$ is non-trivial. 
Proof. It was already discussed above that $\gamma, D$ and $\pi(a), a \in \mathcal{A}$, satisfy the commutation relations of a $\mathbb{Z}_{2}$-graded spectral triple. To prove that commutators the $[D, \pi(a)]$ are bounded, it suffices to consider the generators from $\mathcal{K}_{S}$ and $\mathcal{C}^{\infty}\left(\mathbb{T}_{g, q}\right)$ since, by the Leibniz rule for commutators, $[D, \pi(a b)]=[D, \pi(a)] \pi(b)+\pi(a)[D, \pi(b)]$.

If the operator $A \in \mathcal{K}_{S}$ is given by a matrix of rapid decay $\left(a_{k n}\right)_{k, n \in \mathbb{N}_{0}}$, then the matrices $\left(n a_{k n}\right)_{k, n \in \mathbb{N}_{0}}$ and $\left(k a_{k n}\right)_{k, n \in \mathbb{N}_{0}}$ corresponding to $N A$ and $A N$, respectively, are again matrices of rapid decay and thus define bounded operators belonging to $\mathcal{K}_{S}$. Using the relations $S^{*} N=(N+1) S^{*}$ and $S(N+1)=N S$, we get

$$
[D, \pi(A)]=\left(\begin{array}{cc}
0 & S^{*} N A-A N S^{*}-A S^{*} \\
S N A-A N S+S A & 0
\end{array}\right)
$$

which is bounded because all the operators $A, S, S^{*}, A N$ and $N A$ belong to $B\left(\ell_{2}(\mathbb{N})\right)$. We can conclude even more, namely that the entries of $[D, A]$ belong to $\mathcal{K}_{S}$ since, as easily seen, the product of a matrix of rapid decay with the shift operator or its adjoint yields again a matrix of rapid decay.

Next, let $f \in C^{\infty}\left(\bigvee^{2 g} \mathbb{S}^{1}\right)$ so that $T_{f} \in \mathcal{C}^{\infty}\left(\mathbb{T}_{g, q}\right)$. Then, by (8) and $(9)$,

$$
\begin{aligned}
{\left[S^{*} N, T_{f}\right] e_{n} } & =\sum_{k \in \mathbb{N}_{0}} k f_{k-n} e_{k-1}-\sum_{k \in \mathbb{N}_{0}} n f_{k-n+1} e_{k}=\sum_{k \in \mathbb{N}_{0}}(k-n+1) f_{k-n+1} e_{k} \\
& =\sum_{k \in \mathbb{Z}} k f_{k} S^{\# k+1} e_{n}=-\mathrm{i} T_{\bar{u} f^{\prime}} e_{n}
\end{aligned}
$$

hence

$$
\left[S^{*} N, T_{f}\right]=-\mathrm{i} T_{\bar{u} f^{\prime}} \in \mathcal{T}, \quad\left[N S, T_{f}\right]=\left(-\left[S^{*} N, T_{\bar{f}}\right]\right)^{*}=-\mathrm{i} T_{u f^{\prime}} \in \mathcal{T} .
$$

Therefore $\left[D, \pi\left(T_{f}\right)\right] \in B(\mathcal{H})$ since the entries of this $2 \times 2$-matrix belong to the Toeplitz algebra $\mathcal{T} \subset B\left(\ell_{2}(\mathbb{N})\right)$. This finishes the proof that $[D, \pi(a)] \in B(\mathcal{H})$ for all $a \in \mathcal{A}$.

Furthermore, one readily verifies that the vectors $b_{k}, k \in \mathbb{Z}$, in 15 form a basis of eigenvectors for $\mathcal{H}$ and that $D b_{k}=k b_{k}$. Since each eigenvalue $k \in \mathbb{Z}=\operatorname{spec}(D)$ has multiplicity 1 , the resolvent $(D+\mathrm{i})^{-1}$ is compact and the spectral triple is $1^{+}$-summable.

To prove the regularity of the spectral triple, first note that

$$
\begin{array}{r}
{[|D|, A]=\left(\begin{array}{cc}
{\left[N, A_{11}\right]} & {\left[N, A_{12}\right]+A_{12}} \\
{\left[N, A_{21}\right]-A_{21}} & {\left[N, A_{22}\right]}
\end{array}\right)} \\
\text { for } A=\left(\begin{array}{ll}
A_{11} & A_{12} \\
A_{21} & A_{22}
\end{array}\right) \in B\left(\ell_{2}(\mathbb{N}) \oplus \ell_{2}(\mathbb{N})\right) .
\end{array}
$$

Hence it suffices to show that all elements $a \in \mathcal{A}$ and all entries of $[D, \pi(a)]$ belong to $\bigcap_{n \in \mathbb{N}} \operatorname{dom}\left(\delta_{N}^{n}\right)$, where $\delta_{N}(x):=[N, x]$ for $x \in B\left(\ell_{2}(\mathbb{N})\right)$.

If $A \in \mathcal{K}_{S}$ is given by a rapid decay matrix, then $N^{n} A \in \mathcal{K}_{s}$ and $A N^{n} \in \mathcal{K}_{s}$ for all $n \in \mathbb{N}$ by the very definition of a rapid decay matrix. This implies $\mathcal{K}_{s} \subset \bigcap_{n \in \mathbb{N}} \operatorname{dom}\left(\delta_{N}^{n}\right)$. As concluded below equation $(16)$, all entries of $[D, \pi(A)]$ belong again to $\mathcal{K}_{S}$. Therefore we have $\pi(A),[D, \pi(A)] \in \bigcap_{n \in \mathbb{N}} \operatorname{dom}\left(\delta_{|D|}^{n}\right)$ for all $A \in \mathcal{K}_{S}$.

Let now $f \in C^{\infty}\left(\mathbb{S}^{1}\right)$. As in 17 , we have $\left[N, T_{f}\right] e_{n}=\sum_{k \in \mathbb{N}_{0}}(k-n) f_{k-n} e_{k}$, thus

$$
\delta_{N}^{n}\left(T_{f}\right)=(-\mathrm{i})^{n} T_{f^{(n)}}
$$


by equation (9). Consequently, $T_{f} \in \bigcap_{n \in \mathbb{N}} \operatorname{dom}\left(\delta_{N}^{n}\right)$. As $u f^{\prime}$ and $\bar{u} f^{\prime}$ also belong to $C^{\infty}\left(\mathbb{S}^{1}\right)$, we conclude from the definition of $\mathcal{C}^{\infty}\left(\mathbb{T}_{g, q}\right)$ together with 18 and 19 that $\pi\left(T_{f}\right),\left[D, \pi\left(T_{f}\right)\right] \in \bigcap_{n \in \mathbb{N}} \operatorname{dom}\left(\delta_{|D|}^{n}\right)$ for all $T_{f} \in \mathcal{C}^{\infty}\left(\mathbb{T}_{g, q}\right)$. Since the elements from $\mathcal{K}_{S}$ and $\mathcal{C}^{\infty}\left(\mathbb{T}_{g, q}\right)$ generate $\mathcal{A}$, the regularity follows from the Leibniz rule for commutators.

Having a regular, even spectral triple, we know that its fundamental class $F$ from (14) defines an even Fredholm module. Since $\operatorname{ind}(D)=\operatorname{ind}\left(S^{*}\right)=1 \neq 0$, the fundamental class is non-trivial.

The restriction to $C\left(\mathbb{T}_{g, q}\right)$ was made only for notational convenience. Clearly, we have $\mathcal{K}_{S} \subset C\left(\mathbb{P}_{g, q}\right)$ without any modification. Analyzing the proofs of Proposition 3.1 and Theorem 3.2 one easily realizes that only the differentiability of the function $f$ in the definition of $T_{f} \in \mathcal{C}^{\infty}\left(\mathbb{T}_{g, q}\right)$ was used. So, if we define

$$
C^{\infty}\left(\mathbb{P}_{g, q}\right):=\left\{T_{f} \in C\left(\mathbb{P}_{g, q}\right): f \in C\left(\forall \mathbb{S}^{1}\right) \cup C^{\infty}\left(\mathbb{S}^{1}\right)\right\},
$$

then all arguments in the proofs remain valid. Therefore we can state the analogous results of Theorem 3.2 for $C\left(\mathbb{P}_{g, q}\right)$.

Corollary 3.3. Let $\mathcal{A} \subset C\left(\mathbb{P}_{g, q}\right)$ denote the ${ }^{*}$-algebra generated by the elements of $\mathcal{K}_{S}$ and $C^{\infty}\left(\mathbb{P}_{g, q}\right)$. With the same definitions of $\mathcal{H}, D, \gamma$ and $\pi: \mathcal{A} \rightarrow B(\mathcal{H})$ as in Theorem 3.2 . $(\mathcal{A}, D, \mathcal{H}, \gamma)$ yields a $1^{+}$-summable regular even spectral triple for $C\left(\mathbb{P}_{g, q}\right)$. The spectrum, the eigenvalues and the orthonormal basis of eigenvectors from Theorem 3.2 remain unchanged for the Dirac operator $D$ and its fundamental class is non-trivial.

At the first glance, it might be surprising that there exist Dirac operators on noncommutative versions of a non-orientable manifolds. However, the Dirac operator of a spectral triple should rather be viewed as an analog of an elliptic first order differential operator and not necessarily as the Dirac operator on a spin manifold. Nevertheless, since the Dirac operator is related to topological invariants via the index theorem, it is a strange effect of noncommutative geometry that the same operator can be used for different noncommutative spaces among which some have $K$-groups with torsion.

3.2. Real structure and first order condition. In the context of spin geometry, a real structure singles out those manifolds that admit a real spin structure. The results below will show that our spectral triples cannot be equipped with a real structure in the exact sense. Since this result would not be surprising for a quantum space for which the classical counterpart does not admit a real spin structure, we restrict ourselves in this section to the quantized orientable surfaces $C\left(\mathbb{T}_{g, q}\right)$.

A real structure for a spectral triple $(\mathcal{A}, \mathcal{H}, D)$ is given by an anti-unitary operator $J$ satisfying $J^{2}= \pm$ id and $J D= \pm D J$. For an even spectral triple $(\mathcal{A}, \mathcal{H}, D, \gamma)$, one requires additionally $J \gamma= \pm \gamma J$. The signs depend on the dimension of the underlying quantum space. For instance,

$$
J^{2}=-\mathrm{id}, \quad J D=D J, \quad J \gamma=-\gamma J
$$

in dimension 2 , and

$$
J^{2}=\mathrm{id}, \quad J D=-D J
$$

in dimension 1. 
Given a real structure $J$, one says that the spectral triple satisfies the first order condition if for all $a, b \in \mathcal{A}$

$$
\left[\pi(a), J \pi(b) J^{-1}\right]=0, \quad\left[[D, \pi(a)], J \pi(b) J^{-1}\right]=0 \quad \text { for all } a, b \in \mathcal{A} .
$$

It was observed in [7] and [8] that, under certain circumstances, a real structure might not exist for quantized real spin manifolds and it was proposed to modify the first order condition 23 by requiring only

$$
\left[\pi(a), J \pi(b) J^{-1}\right] \in \mathcal{K}_{S}(\mathcal{H}), \quad\left[[D, \pi(a)], J \pi(b) J^{-1}\right] \in \mathcal{K}_{S}(\mathcal{H}) \quad \text { for all } a, b \in \mathcal{A},
$$

where $\mathcal{K}_{S}(\mathcal{H}) \subset B(\mathcal{H})$ denotes the ideal of matrices of rapid decay (associated to an orthonormal basis). Here we assume that $\mathcal{H}$ is separable. In the context of noncommutative geometry, the matrices of rapid decay are considered as infinitesimals of arbitrary high order.

The first result of this section shows that the spectral triples from Theorem 3.2 do not admit a real structure in the exact sense.

Proposition 3.4. Let $(\mathcal{A}, \mathcal{H}, D, \gamma)$ denote the spectral triple described in Theorem 3.2 . Then there does not exist an anti-unitary operator $J$ on $\mathcal{H}$ satisfying (21).

Proof. From $J^{-1} D J e_{k}=D e_{k}=k e_{k}$, it follows that $D J e_{k}=k J e_{k}$, hence $J e_{k}=\alpha_{k} e_{k}$, where $\alpha_{k} \in \mathbb{C}$. Since $J$ is anti-unitary, we have necessarily $\left|\alpha_{k}\right|=1$, and thus

$$
J^{2} e_{n}=J\left(\alpha_{k} e_{k}\right)=\bar{\alpha}_{k} J e_{k}=\bar{\alpha}_{k} \alpha_{k} e_{k}=e_{k},
$$

which contradicts the first equation of (21).

Ignoring for a moment the commutation relations $(21)$ and $(22)$, it is also impossible to find an anti-unitary operator satisfying 23 .

Proposition 3.5. Let $\mathcal{A}$ denote the ${ }^{*}$-algebra from Proposition 3.1 and $\pi: \mathcal{A} \rightarrow B(\mathcal{H})$ the representation from Theorem 3.2 . Then there does not exist an anti-unitary operator $J$ on $\mathcal{H}$ satisfying the first order condition 23 .

Proof. Recall that $\mathcal{H}=\ell_{2}(\mathbb{N}) \oplus \ell_{2}(\mathbb{N})$ and $\pi(a)\left(v_{+} \oplus v_{-}\right):=a v_{+} \oplus a v_{-}$. If an operator $A=\left(\begin{array}{ll}A_{11} & A_{12} \\ A_{21} & A_{22}\end{array}\right) \in B\left(\ell_{2}(\mathbb{N}) \oplus \ell_{2}(\mathbb{N})\right)$ commutes with $\pi(a)$ for all $a \in \mathcal{A}$, then we have necessarily $\left[k, A_{i j}\right]=0$ for all $k \in \mathcal{K}_{S}$ and $i, j=1,2$. Since $\mathcal{K}_{S}$ is dense in $K\left(\ell_{2}(\mathbb{N})\right)$, it follows that $A_{i j}=c_{i j}$ id with $c_{i j} \in \mathbb{C}$. Therefore we get for any anti-unitary operator $J$ satisfying 23

$$
\operatorname{dim}\{A \in B(\mathcal{H}):[\pi(a), A]=0 \text { for all } a \in \mathcal{A}\}=4 \geq \operatorname{dim}\left\{J \pi(b) J^{-1}: b \in \mathcal{A}\right\}=\infty,
$$

which is a contradiction.

If one wants to allow for the existence of a real structure despite the negative result of Proposition 3.5. one may consider to weaken the first order condition 23 by requiring only the modified version 24. But then the problem of Proposition 3.4 still persists. However, the problem was caused by taking the commutation relations 21 of a spectral triple of dimension 2. On the other hand, our spectral triples are $1^{+}$-summable, thus their metric dimension is 1 instead of 2. Under the requirements of equations (22) and (24), we can prove the following positive result. 
Proposition 3.6. Let $(\mathcal{A}, \mathcal{H}, D, \gamma)$ denote the spectral triple given in Theorem 3.2, and let $\left\{b_{k}: k \in \mathbb{Z}\right\}$ be the orthonormal basis defined in $(15)$. Then the anti-unitary operator $J$ given by

$$
J b_{k}=b_{-k}, \quad k \in \mathbb{Z},
$$

satisfies the conditions (22) and (24).

Proof. Since $b_{k}$ is an eigenvector of $D$ corresponding to the eigenvalue $k \in \mathbb{Z}$, we have $J D J^{-1} b_{k}=-k b_{k}=-D b_{k}$, thus $J D=-D J$. Obviously, $J^{2}=1$, so 22 is satisfied.

If $b \in \mathcal{K}_{S}$, then $J \pi(b) J^{-1} \in \mathcal{K}_{S}(\mathcal{H})$ and therefore 24 holds for all $a \in \mathcal{A}$ since $\mathcal{K}_{S}(\mathcal{H})$ is an ideal in $B(\mathcal{H})$. Similarly, if $a \in \mathcal{K}_{S}$, then, as in the proof of Theorem 3.2 $N a, a N \in \mathcal{K}_{S}$. Therefore $[D, \pi(a)] \in \mathcal{K}_{S}(\mathcal{H})$, and since also $\pi(a) \in \mathcal{K}_{S}(\mathcal{H})$, the condition (24) is now fulfilled for all $b \in \mathcal{A}$.

It remains to show that 24 holds for $a, b \in \mathcal{C}^{\infty}\left(\mathbb{T}_{g, q}\right)$. So, let $a=T_{g}$ and $b=T_{f}$, where $g, f \in C^{\infty}\left(\bigvee^{2 g} \mathbb{S}^{1}\right)$. From (15) and (25), we get $J\left(e_{k} \oplus e_{n}\right)=\left(-e_{k}\right) \oplus e_{n}$ for all $k, n \in \mathbb{N}_{0}$. Writing $T_{f}=\sum_{k \in \mathbb{Z}} f_{k} S^{\# k}$ as in (8) and setting $e_{n}^{+}:=e_{n} \oplus 0$ and $e_{n}^{-}:=0 \oplus e_{n}$, we compute

$$
J \pi\left(T_{f}\right) J^{-1} e_{n}^{ \pm}=\sum_{k \in \mathbb{N}_{0}} \bar{f}_{k-n} e_{k}^{ \pm}=\sum_{k \in \mathbb{Z}} \bar{f}_{k} S^{\# k} e_{n}^{ \pm}=\pi\left(T_{\hat{f}}\right) e_{n}^{ \pm},
$$

where $\hat{f} \in C^{\infty}\left(\mathbb{S}^{1}\right)$ is defined by $\hat{f}(z):=\overline{f(\bar{z})}$. Therefore, by 13, 18) and 26,

$$
\begin{gathered}
{\left[\pi\left(T_{g}\right), J \pi\left(T_{f}\right) J^{-1}\right]=\pi\left(\left[T_{g}, T_{\hat{f}}\right]\right)} \\
{\left[\left[D, \pi\left(T_{g}\right)\right], J \pi\left(T_{f}\right) J^{-1}\right]=-\mathrm{i}\left(\begin{array}{cc}
0 & {\left[T_{\bar{u} g^{\prime}}, T_{\hat{f}}\right]} \\
{\left[T_{u g^{\prime}}, T_{\hat{f}}\right]} & 0
\end{array}\right) .}
\end{gathered}
$$

Since $g, \hat{f}, u g^{\prime}, \bar{u} g^{\prime} \in C^{\infty}\left(\mathbb{S}^{1}\right)$, and hence its Fourier coefficients are sequences of rapid decay, we conclude as in the paragraph with Equation (11) that the commutators $\left[T_{g}, T_{\hat{f}}\right]$, $\left[T_{\bar{u} g^{\prime}}, T_{\hat{f}}\right]$ and $\left[T_{u g^{\prime}}, T_{\hat{f}}\right]$ belong to $\mathcal{K}_{S}$. From this, the result follows.

3.3. Finiteness. We say that a spectral triple $(\mathcal{A}, \mathcal{H}, D)$ satisfies the finiteness condition, if there exists a dense subset of "smooth" vectors $\mathcal{H}_{\infty} \subset \bigcap_{n \in \mathbb{N}} \operatorname{dom}\left(D^{n}\right)$ which is isomorphic to a finitely generated left $\mathcal{A}$-projective module. This finitely generated projective module is considered as a module of smooth sections of the vector bundle on which the Dirac operator acts (e.g. spin bundle). If $\mathcal{H}_{\infty}$ is a core for the self-adjoint operator $D$, then the Dirac operator $D$ is uniquely determined by its restriction to $\mathcal{H}_{\infty}$.

Stable isomorphism classes of finitely generated projective modules of a (pre-) $\mathrm{C}^{*}$-algebra are classified by the associated $K_{0}$-group. Recall from Section 2 that a non-trivial generator of $K_{0}\left(C\left(\mathbb{T}_{g, q}\right)\right)$ is given by the 1-dimensional projection $p_{e_{0}}=1-S S^{*} \in \mathcal{K}_{S} \subset \mathcal{A}$. Consider now the finitely generated left $\mathcal{A}$-projective module $\mathcal{A}_{\infty}:=\mathcal{A}\left(1-S S^{*}\right) \subset$ $B\left(\ell_{2}(\mathbb{N})\right)$ together with the vector state and the non-negative sesquilinear form

$$
\psi_{0}(a):=\left\langle e_{0}, a e_{0}\right\rangle, \quad\langle\langle b, a\rangle\rangle:=\psi_{0}\left(b^{*} a\right)=\left\langle b e_{0}, a e_{0}\right\rangle, \quad a, b \in \mathcal{A}\left(1-S S^{*}\right) .
$$

The next proposition shows that $\bigcap_{n \in \mathbb{N}} \operatorname{dom}\left(D^{n}\right) \cong \mathcal{A}\left(1-S S^{*}\right) \oplus \mathcal{A}\left(1-S S^{*}\right)$ satisfying thus the finiteness condition. We will give the proof for $C\left(\mathbb{T}_{g, q}\right)$ but the statement holds also for $\mathcal{A} \subset C\left(\mathbb{P}_{g, q}\right)$. 
Proposition 3.7. Consider the finitely generated left $\mathcal{A}$-module $\mathcal{A}\left(1-S S^{*}\right)$ together with the sesquilinear form given in 27). Then the left $\mathcal{A}$-modules $\mathcal{A}\left(1-S S^{*}\right) \oplus \mathcal{A}\left(1-S S^{*}\right)$ and $\mathcal{H}_{\infty}:=\bigcap_{n \in \mathbb{N}} \operatorname{dom}\left(D^{n}\right)$ are isometrically isomorphic.

Proof. Since $\bigcap_{n \in \mathbb{N}} \operatorname{dom}\left(D^{n}\right)=\bigcap_{n \in \mathbb{N}} \operatorname{dom}\left(N^{n}\right) \oplus \bigcap_{n \in \mathbb{N}} \operatorname{dom}\left(N^{n}\right)$ with the diagonal $\mathcal{A}$-action, it suffices to show that $\mathcal{A}\left(1-S S^{*}\right) \cong \bigcap_{n \in \mathbb{N}} \operatorname{dom}\left(N^{n}\right)$. Note that

$$
\begin{aligned}
\bigcap_{n \in \mathbb{N}} \operatorname{dom}\left(N^{n}\right) & =\left\{\sum_{k \in \mathbb{N}_{0}} \alpha_{k} \mathrm{e}_{k} \in \ell_{2}(\mathbb{N}): \sum_{n \in \mathbb{N}_{0}}\left|\alpha_{k}\right|^{2} k^{2 n}<\infty\right\} \\
& =\left\{\sum_{k \in \mathbb{N}_{0}} \alpha_{k} \mathrm{e}_{k} \in \ell_{2}(\mathbb{N}):\left(\alpha_{k}\right)_{k \in \mathbb{N}_{0}} \in \mathcal{S}(\mathbb{N})\right\}
\end{aligned}
$$

where $\mathcal{S}(\mathbb{N})$ denotes the space of sequences of rapid decay. We claim that

$$
\Phi: \mathcal{A}\left(1-S S^{*}\right) \subset B\left(\ell_{2}(\mathbb{N})\right) \longrightarrow \bigcap_{n \in \mathbb{N}} \operatorname{dom}\left(N^{n}\right), \quad \Phi(x):=x e_{0},
$$

defines an isometric isomorphism.

To see that $\Phi$ is well defined, suppose that $T_{f} \in \mathcal{C}^{\infty}\left(\mathbb{T}_{g, q}\right)$, where $f \in C^{\infty}\left(\bigvee^{2 g} \mathbb{S}^{1}\right)$, and write $f=\sum_{k \in \mathbb{Z}} f_{k} u^{k}$ in its Fourier series expansion. From equation (8), we get $\Phi\left(T_{f}\left(1-S S^{*}\right)\right)=\sum_{k \in \mathbb{N}_{0}} f_{k} e_{k}$. Since $f \in C^{\infty}\left(\mathbb{S}^{1}\right)$, the sequence $\left(f_{k}\right)_{n \in \mathbb{N}_{0}}$ belongs to $\mathcal{S}(\mathbb{N})$ (cf. Section 3.1). Therefore, by [28), $\Phi\left(T_{f}\left(1-S S^{*}\right)\right) \in \bigcap_{n \in \mathbb{N}} \operatorname{dom}\left(N^{n}\right)$. If $a \in \mathcal{K}_{S}$ and $\left(a_{k j}\right)_{k, j \in \mathbb{N}_{0}}$ denotes the corresponding matrix of rapid decay, then $\left(a_{k 0}\right)_{k \in \mathbb{N}_{0}} \in \mathcal{S}(\mathbb{N})$ and therefore $\Phi\left(a\left(1-S S^{*}\right)\right)=\sum_{k \in \mathbb{N}_{0}} a_{k 0} e_{k} \in \bigcap_{n \in \mathbb{N}} \operatorname{dom}\left(N^{n}\right)$. Since each element from $a \in \mathcal{A}$ can be written as $a=\left(a-T_{\sigma(a)}\right)+T_{\sigma(a)} \in \mathcal{K}_{S}+\mathcal{C}^{\infty}\left(\mathbb{T}_{g, q}\right)$ it follows that $\Phi\left(\mathcal{A}\left(1-S S^{*}\right)\right) \subset \bigcap_{n \in \mathbb{N}} \operatorname{dom}\left(N^{n}\right)$.

Clearly, $\Phi$ is left $\mathcal{A}$-linear since $\mathcal{A} \subset B\left(\ell_{2}(\mathbb{N})\right)$. To show the surjectivity of $\Phi$, let $v=\sum_{k \in \mathbb{N}_{0}} \alpha_{k} \mathrm{e}_{k} \in \bigcap_{n \in \mathbb{N}} \operatorname{dom}\left(N^{n}\right)$. Then $a:=\left(\alpha_{k} \delta_{0, j}\right)_{k, j \in \mathbb{N}_{0}} \in \mathcal{K}_{S}$, where $\delta_{i, j}$ denotes the Kronecker delta, and $\Phi\left(a\left(1-S S^{*}\right)\right)=v$. Moreover, $\Phi$ is injective. To see this, assume that $\Phi\left(a\left(1-S S^{*}\right)\right)=0$. Then $a\left(1-S S^{*}\right) e_{0}=\Phi\left(a\left(1-S S^{*}\right)\right)=0$ and $a\left(1-S S^{*}\right) e_{k}=0$ for all $k \neq 0$ since $1-S S^{*}$ is the orthogonal projection onto $\mathbb{C} e_{0}$. Thus $a\left(1-S S^{*}\right)=0$ in $\mathcal{A}\left(1-S S^{*}\right) \subset B\left(\ell_{2}(\mathbb{N})\right)$. So we just proved that the map $\Phi$ in 29 defines an isomorphism of left $\mathcal{A}$-modules.

It remains to show that $\Phi$ is actually an isometry. From (27) and 29], we get

$$
\left\langle\Phi\left(a\left(1-S S^{*}\right)\right), \Phi\left(a\left(1-S S^{*}\right)\right)\right\rangle=\left\langle a e_{0}, a e_{0}\right\rangle=\langle\langle a, a\rangle\rangle
$$

for all $a \in \mathcal{A}$. This completes the proof.

Applying the previous proposition to the noncommutative torus $C\left(\mathbb{T}_{1, q}\right)$, we see that the "spin bundle" is given by $\mathcal{A}\left(1-S S^{*}\right) \oplus \mathcal{A}\left(1-S S^{*}\right)$ rather than $\mathcal{A} \oplus \mathcal{A}$ as it should have been in analogy to the classical case. Moreover, by extending the isomorphism $\Phi$ in 29 to its closure, the Hilbert space $\overline{\mathcal{A}\left(1-S S^{*}\right)} \cong \ell_{2}(\mathbb{N}) \subset L_{2}\left(\mathbb{S}^{1}\right)$ looks rather like functions on the circle $\mathbb{S}$ than on the disc $\mathbb{D}$. This observation is in line with the metric dimension 1. It seems that the interior of the quantum disc $K\left(\ell_{2}(\mathbb{N})\right)=\operatorname{ker}(\sigma)$ has the dimension of a (fuzzy) point. Also (18) indicates that the action of the Dirac operator is essentially given by a derivation on the circle. 
3.4. Existence of a volume form or orientation. By a volume form we mean a Hochschild $n$-cycle $\omega$, i.e.,

$$
\begin{aligned}
\omega & =\sum_{j} a_{0 j} \otimes b_{j} \otimes a_{1 j} \otimes \ldots \otimes a_{n j} \in \mathcal{A} \otimes \mathcal{A}^{\mathrm{op}} \otimes \mathcal{A} \otimes \ldots \otimes \mathcal{A}, \\
0 & =\delta_{n}(\omega):=\sum_{j} a_{0 j} \otimes\left(b_{j} a_{1 j}\right) \otimes a_{2 j} \otimes \ldots \otimes a_{n j} \\
& +\sum_{k=1}^{n-1} \sum_{j}(-1)^{k} a_{0 j} \otimes b_{j} \otimes \ldots \otimes a_{k j} a_{k+1, j} \otimes \ldots \otimes a_{n j} \pm \sum_{j} a_{n j} a_{0 j} \otimes b_{j} \otimes \ldots \otimes a_{n-1, j},
\end{aligned}
$$

satisfying

$$
\gamma=\pi_{D}(\omega):=\sum_{j} a_{0 j} J b_{j}^{*} J^{-1}\left[D, a_{1 j}\right] \cdots\left[D, a_{n j}\right],
$$

where $n$ depends on the (metric) dimension of the spectral triple.

As pointed out in the paragraph preceding Proposition 3.6 the metric dimension of our spectral triples is 1. However, for a Hochschild 1-cycle $\omega$, the expression $\pi_{D}(\omega)$ in (30) is an odd operator whereas $\gamma$ is a diagonal operator, so equation 30 cannot hold. Assuming that our quantum surfaces have dimension 2, we face the problem of the non-existence of the real structure $J$, see Proposition 3.4. Unfortunately the problem goes deeper and cannot be resolved in any other way.

Proposition 3.8. For all spectral triples from Section 3.1 and any anti-unitary operator $J$, there does not exist a Hochschild n-cycle satisfying (30).

Proof. For $n$ odd, the same reasoning as in the case $n=1$ applies: Since $D$ is odd, the right hand side of 30 would yield an odd operator whereas $\gamma$ is even, a contradiction.

Assume now that there exist an anti-unitary operator $J$ and a Hochschild $2 k$-cycle $\omega=\sum_{j} a_{0 j} \otimes b_{j} \otimes a_{1 j} \otimes \ldots \otimes a_{2 k, j}$ satisfying $(30)$. Let $\hat{\sigma}: B\left(\ell_{2}(\mathbb{N})\right) \rightarrow B\left(\ell_{2}(\mathbb{N})\right) / K\left(\ell_{2}(\mathbb{N})\right)$ denote the canonical projection and observe that the restriction of $\hat{\sigma}$ to the Toeplitz algebra yields the symbol map. Recall that $N k, k N \in \mathcal{K}_{S}$ for all $k \in \mathcal{K}_{S}$. From (18), we get for all $a \in \mathcal{A}$

$\hat{\sigma}\left(\left[S^{*} N, a\right]\right)=\hat{\sigma}\left(\left[S^{*} N, a-T_{\sigma(a)}+T_{\sigma(a)}\right]\right)=\hat{\sigma}\left(\left[S^{*} N, T_{\sigma(a)}\right]\right)=\hat{\sigma}\left(-\mathrm{i} T_{\bar{u} \sigma(a)^{\prime}}\right)=-\mathrm{i} \bar{u} \sigma(a)^{\prime}$, and similarly $\hat{\sigma}([N S, a])=-\mathrm{i} u \sigma(a)^{\prime}$. Using the facts that the operators $\sigma(t), t \in \mathcal{T}$, commute and that $u \bar{u}=1$, and applying $\hat{\sigma}$ to two consecutive commutators in (30), we obtain

$$
\begin{aligned}
\hat{\sigma}\left(\left[D, a_{2 i, j}\right]\left[D, a_{2 i+1, j}\right]\right) & =\left(\begin{array}{cc}
\hat{\sigma}\left(\left[S^{*} N, a_{2 i, j}\right]\left[N S, a_{2 i+1, j}\right]\right) & 0 \\
0 & \hat{\sigma}\left(\left[N S, a_{2 i, j}\right]\left[S^{*} N, a_{2 i+1, j}\right]\right)
\end{array}\right) \\
& =\left(\begin{array}{cc}
-\sigma\left(a_{2 i, j}\right)^{\prime} \sigma\left(a_{2 i+1, j}\right)^{\prime} & 0 \\
0 & -\sigma\left(a_{2 i, j}\right)^{\prime} \sigma\left(a_{2 i+1, j}\right)^{\prime}
\end{array}\right) .
\end{aligned}
$$

Note that the diagonal entries coincide. This remains true for the right hand side of 30 if $n$ is even. On the other hand, the diagonal entries of $\gamma$ differ by a minus sign. Therefore applying $\hat{\sigma}$ to the diagonal elements of (30) and equating gives

$$
1=\sum_{j}(-1)^{k} \hat{\sigma}\left(a_{0 j} J b_{j}^{*} J^{-1}\right) \sigma\left(a_{1, j}\right)^{\prime} \sigma\left(a_{2, j}\right)^{\prime} \cdots \sigma\left(a_{2 k-1, j}\right)^{\prime} \sigma\left(a_{2 k, j}\right)^{\prime}=-1,
$$

which is a contradiction. 
Observe that the problem of Proposition 3.8 cannot be solved by changing $\gamma$ as in [14] as long as the diagonal entries of $\gamma$ do not coincide.

3.5. Poincaré duality. We mentioned already in Section 3.1 that regular spectral triples give rise to index pairings. To be more precise, one can show that, for an even regular spectral triple $(\mathcal{A}, \mathcal{H}, D, \gamma)$ and a projection $P=P^{2}=P^{*} \in \operatorname{Mat}_{n \times n}(\mathcal{A})$, the map $\pi(P) D_{+-} \pi(P): \pi(P) \mathcal{H}_{-}^{n} \rightarrow \pi(P) \mathcal{H}_{+}^{n}$ yields a Fredholm operator and its index does not depend on the $K_{0}$-class of $P$. Here, $D_{+-}$denotes the upper right corner of the odd operator $D$. Unfortunately, the formulation of Poincaré duality involves a real structure $J$. One says that an even spectral triple with real structure $J$ satisfies Poincaré duality [3], if

$$
\begin{aligned}
K_{0}(\mathcal{A}) \times K_{0}(\mathcal{A}) \ni & ([P],[Q]) \\
& \longmapsto \operatorname{ind}\left(\left(\pi(P) \otimes J \pi(Q) J^{-1}\right) D_{+-}\left(\pi(P) \otimes J \pi(Q) J^{-1}\right)\right) \in \mathbb{Z}
\end{aligned}
$$

defines a non-degenerate pairing, where $P \in \operatorname{Mat}_{n \times n}(\mathcal{A})$ and $Q \in \operatorname{Mat}_{k \times k}(\mathcal{A})$ are projections and the operator in $(31)$ acts between the Hilbert spaces $\left(\pi(P) \otimes J \pi(Q) J^{-1}\right) \mathcal{H}_{-}^{n k}$ and $\left(\pi(P) \otimes J \pi(Q) J^{-1}\right) \mathcal{H}_{+}^{n k}$.

The aim of this section is to explain that the Poincaré duality fails for our spectral triple. The statement does not seem to make much sense because of the non-existence of a real structure proven in Section 3.2 However, the main problem is not the real structure. The next proposition will show that the fundamental class of the Dirac operator only detects the rank of the trivial $K_{0}$-classes and leads to a zero pairing with $K_{0^{-}}$ classes represented by compact operators. Let us recall here from [15] (see also the end of Section 2 that $K_{0}\left(C\left(\mathbb{T}_{g, q}\right)\right) \cong \mathbb{Z} \oplus \mathbb{Z} \cong K_{0}\left(K\left(\ell_{2}(\mathbb{N})\right)\right) \oplus K_{0}(\mathbb{C})$.

Proposition 3.9. Let $(\mathcal{A}, \mathcal{H}, D, \gamma)$ denote the spectral triple from Theorem 3.2. For any odd or even anti-unitary operator $J$ on $\mathcal{H}=\ell_{2}(\mathbb{N}) \oplus \ell_{2}(\mathbb{N})$, the index pairing (31) (if well defined) is degenerate.

Proof. Let $p_{e_{0}}$ denote the 1-dimensional projection onto $\mathbb{C} e_{0} \subset \mathcal{H}_{-}=\ell_{2}(\mathbb{N})$. Then $\left[p_{e_{0}}\right] \in$ $K_{0}\left(C\left(\mathbb{T}_{g, q}\right)\right)$, and for any projection $Q:=\pi\left(Q_{0}\right)$ with $\left[Q_{0}\right] \in K_{0}\left(C\left(\mathbb{T}_{g, q}\right)\right)$, we have

$$
\operatorname{dim}\left(\left(p_{e_{0}} \otimes J Q J^{-1}\right) \mathcal{H}_{-}^{n}\right)=\operatorname{dim}\left(J Q J^{-1}\left(\left(p_{e_{0}} \ell_{2}(\mathbb{N})\right) \otimes \mathbb{C}^{n}\right)\right) \leq \operatorname{dim}\left(\mathbb{C} \otimes \mathbb{C}^{n}\right)=n<\infty .
$$

Hence the operator

$$
\left(p_{e_{0}} \otimes J Q J^{-1}\right) S^{*} N\left(p_{e_{0}} \otimes J Q J^{-1}\right):\left(p_{e_{0}} \otimes J Q J^{-1}\right) \ell_{2}(\mathbb{N})^{n} \rightarrow\left(p_{e_{0}} \otimes J Q J^{-1}\right) \ell_{2}(\mathbb{N})^{n}
$$

acts between finite dimensional spaces and therefore its index is always 0 .

Note that the problem arises because the compact operators $K\left(\ell_{2}(\mathbb{N})\right) \subset C\left(\mathbb{T}_{g, q}\right)$ act on $\mathcal{H}_{-}=\ell_{2}(\mathbb{N})$ by the identity and thus $\operatorname{dim}\left(p_{e_{0}} \mathcal{H}_{-}\right)=1<\infty$. So we arrive again at the conclusion that the Hilbert space $\mathcal{H}=\ell_{2}(\mathbb{N}) \oplus \ell_{2}(\mathbb{N})$ is "too small" for a non-trivial index pairing.

Acknowledgments. This work was supported by CIC-UMSNH and is part of the international project "Quantum Dynamics" supported by EU grant H2020-MSCA-RISE2015-691246 and co-financed by Polish Government grant W2/H2020/2016 awarded for the years 2016-2019. 


\section{References}

[1] T. Brzeziński, N. Ciccoli, L. Dąbrowski, A. Sitarz, Twisted reality condition for Dirac operators, Math. Phys. Anal. Geom. 19 (2016), art. 16.

[2] A. Connes, Noncommutative Geometry, Academic Press, San Diego, 1994.

[3] A. Connes, Noncommutative geometry and reality, J. Math. Phys. 36 (1995), 6194-6231.

[4] A. Connes, Gravity coupled with matter and the foundations of noncommutative geometry, Comm. Math. Phys. 182 (1996), 155-176.

[5] A. Connes, Cyclic cohomology, quantum group symmetries and the local index formula for $S U_{q}(2)$, J. Inst. Math. Jussieu 3 (2004), 17-68.

[6] A. Connes, G. Landi, Noncommutative manifolds, the instanton algebra and isospectral deformations, Comm. Math. Phys. 221 (2001), 141-159.

[7] L. Dąbrowski, G. Landi, M. Paschke, A. Sitarz, The spectral geometry of the equatorial Podleś sphere, C. R. Acad. Sci. Paris, Ser. I 340 (2005), 819-822.

[8] L. Dąbrowski, G. Landi, A. Sitarz, W. van Suijlekom, J. C. Várilly, The Dirac operator on $S U_{q}(2)$, Comm. Math. Phys. 259 (2005), 729-759.

[9] L. Dąbrowski, A. Sitarz, Dirac operator on the standard Podleś quantum sphere, in: Noncommutative Geometry and Quantum Groups, Banach Center Publ. 61, Polish Acad. Sci. Inst. Math., Warsaw, 2003, 49-58.

[10] F. Díaz, E. Wagner, Dirac operator on a noncommutative Toeplitz torus, in: Geometric Methods in Physics XXXVI, Trends Math., Birkhäuser/Springer, Cham, 2019, 103-109.

[11] J. M. Gracia-Bondía, J. C. Várilly, H. Figueroa, Elements of Noncommutative Geometry, Birkhäuser, Boston, 2001.

[12] S. Klimek, A. Lesniewski, A two-parameter quantum deformation of the unit disc, J. Funct. Anal. 115 (1993), 1-23.

[13] N. L. Vasilevski, Commutative algebras of Toeplitz operators on the Bergman space, Oper. Theory Adv. Appl. 185, Birkhäuser, Basel, 2008.

[14] E. Wagner, On the noncommutative spin geometry of the standard Podles sphere and index computations, J. Geom. Phys. 59 (2009), 998-1016.

[15] E. Wagner, Compact quantum surfaces of any genus, in preparation. 